Yogesh Kumar Vijaywar, N. P. S. Bawa, Praveen Kumar Shrivastava

\title{
COINCIDENCE AND COMMON FIXED POINT THEOREMS FOR HYBRID CONTRACTIONS IN SYMMETRIC SPACES
}

\begin{abstract}
Using compatibility of type $(N)$ and $(\mathrm{E}-\mathrm{A})$ property of hybrid pair of mappings we obtain some new coincidence and common fixed point theorems under strict contractive conditions in symmetric (semi-metric) space.
\end{abstract}

\section{Introduction}

Amari and E. L. Montawakil [1], [2] introduced (E-A) property and obtain some relatively more general common fixed point theorems for strict contractive conditions in metric space. Imdad at al. [4] and Cho et al. [3] obtained some coincidence and fixed point theorems in symmetric (semimetric) space, under strict contractions using (E-A) property. Kamran [8] obtained some coincidence and fixed point theorems by extending this (E-A) property for pair of single-valued and multi valued mappings i.e. for hybrid pair of mappings. On the other hand Shrivastava, Bawa and Singh [14] have introduced the notion of compatibility of type $(N)$ which is a generalization of compatibility and weak compatibility of hybrid pairs.

The aim of this paper is to obtain some new coincidence and common fixed point theorems under strict contractions in symmetric (semi-metric) spaces using compatibility of type $(N)$ hybrid pair.

\section{Preliminaries}

Definition 1. A symmetric on a set $X$ is a function $d: X \times X \rightarrow[0, \infty)$ such that for all $x, y \in X$

(i) $d(x, y)=0 \Leftrightarrow x=y$

(ii) $d(x, y)=d(y, x)$.

2000 Mathematics Subject Classification: Primary 47H10; Secondary 54H25.

Key words and phrases: symmetric space, semi-metric space, (E-A)-property, compatibility of type $(\mathrm{N})$, coincident point, common fixed point. 
If $d$ is symmetric on a set $X$, then for $x \in X$ and $\varepsilon>0$, we write $S(x, \varepsilon)=\{y \in X: d(x, y)<\varepsilon\}$. A topology $\tau(d)$ on $X$ is given by $U \in \tau(d)$ if and only if for each $x \in X, B(x, \varepsilon) \subset U$ for some $\varepsilon>0$. A set $S \subset X$ is a neighborhood of $b \in X$ iff there exists $U \in \tau(d)$ such that $b \in U \subset S$. A symmetric $d$ is a semi-metric if for each $x \in X$ and for each $\varepsilon>0, B(x, \varepsilon)$ is a neighborhood of $x$ in topology $\tau(d)$. Note that $\lim _{n \rightarrow \infty} d\left(x_{n}, x\right)=0$ iff $x_{n} \rightarrow x$ in the topology $\tau(d)$.

Definition 2. A semi-metric space $X$ is a topological space whose topology $\tau(d)$ on $X$ is induced by semi-metric $d$. In what follows symmetric space as well as semi-metric space will be denoted by $(X, d)$.

The distinction between a symmetric and a semi-metric is evident as one can easily construct a symmetric $d$ such that $S(x, \varepsilon)$ need not be a neighborhood of $x$ in $\tau(d)$. For a symmetric $d$ on $X$ the following two axioms were given by Wilson [15]:

[W3] Given $\left\{x_{n}\right\}, x$ and $y$ in $X, d\left(x_{n}, x\right) \rightarrow 0$ and $d\left(x_{n}, y\right) \rightarrow 0$ imply that $x=y$.

[W4] Given $\left\{x_{n}\right\},\left\{y_{n}\right\}$ and $x$ in $X, d\left(x_{n}, x\right) \rightarrow 0$ and $d\left(x_{n}, y_{n}\right) \rightarrow 0$ imply that $d\left(y_{n}, x\right) \rightarrow 0$.

Here it may be noted that for a semi-metric $d$ if $\tau(d)$ is Hausdorff, then [W3] holds.

Now we state some definitions:

Definition 3. ([4], [8]) Let $X$ be a symmetric (semi-metric) space with symmetric $d$. Then, for $x \in X$ and $A \subset X, d(x, A)=\inf \{d(x, y): y \in A\}$.

We denote by $C B(X)$ the class of all non empty bounded closed subsets of $X$.

Let $H$ be Hausdorff symmetric with respect to $d$, i.e.

$$
H(A, B)=\max \left\{\sup _{x \in A} d(x, B), \sup _{x \in B} d(y, A)\right\} \forall A, B \in C B(X) .
$$

Definition 4. ([4], [8]) Let $(X, d)$ be a symmetric (semi-metric) space. Then maps $f: X \rightarrow X$ and $F: X \rightarrow C B(X)$ are said to be compatible if $f F x \in C B(X)$ for all $x \in X$ and $H\left(f F x_{n}, F f x_{n}\right) \rightarrow 0$ whenever $\left\{x_{n}\right\}$ is a sequence in $X$ such that $F x_{n} \rightarrow A \in C B(X)$ and $f x_{n} \rightarrow t \in A$.

Definition 5. ([4], [8]) Let $(X, d)$ be a symmetric (semi-metric) space. Then maps $f: X \rightarrow X$ and $F: X \rightarrow C B(X)$ are said to be non compatible if $f F x \in C B(X)$ for all $x \in X$ and there exists at least one sequence $\left\{x_{n}\right\}$ in $X$ such that $F x_{n} \rightarrow A \in C B(X)$ and $f x_{n} \rightarrow t \in A$. But $\lim _{n \rightarrow \infty} H\left(f F x_{n}, F f x_{n}\right)$ is either non zero or non existent. 
Definition 6. ([4], [8]) Let $(X, d)$ be a symmetric (semi-metric) space. Then maps $f: X \rightarrow X$ and $F: X \rightarrow C B(X)$ are said to be weakly compatible if they commute at their coincidence points. i.e. $f F x=F f x$ whenever $f x \in F x$.

Definition 7. ([3], [5], [12]) Let $(X, d)$ be a symmetric (semi-metric) space. Then maps $f: X \rightarrow X$ and $F: X \rightarrow C B(X)$ are said to be $R$-weakly commuting if, for given $x \in X, f F x \in C B(X)$, and there exists some positive real number $R$ such that $H(f F x, F f x) \leq R d(f x, F x)$.

Definition 8. ([1], [3], [5]) Let $(X, d)$ be a symmetric (semi-metric) space. Then maps $f: X \rightarrow X$ and $F: X \rightarrow C B(X)$ are said to satisfy the property (E-A) if there exists a sequence $\left\{x_{n}\right\}$ in $X$, for some $t \in X$ and $A \in C B(X)$ such that

$$
\lim _{n \rightarrow \infty} f x_{n}=t \in A=\lim _{n \rightarrow \infty} F x_{n} .
$$

EXAmple 1. Consider $X=[1, \infty)$ with the symmetric $d(x, y)=(x-y)^{2}$, define $f: X \rightarrow X$ and $F: X \rightarrow C B(X)$ such that $f x=x+1$ and $F x=[1, x+2]$ for all $x \in X$.

Clearly $X$ is symmetric because

$$
1=d(1,2)>\frac{1}{4}+\frac{1}{4}=d\left(1, \frac{3}{2}\right)+d\left(\frac{3}{2}, 2\right) .
$$

Further consider the sequence $\left\{x_{n}\right\}=\left\{\frac{1}{n}\right\}$. Then clearly $\lim _{n \rightarrow \infty} f x_{n}=1 \in$ $[1,2]=\lim _{n \rightarrow \infty} F x_{n}$. Thus $f$ and $F$ satisfy property (E-A).

EXAMPLE 2. Let $X=[2, \infty)$ be a symmetric space with metric $d(x, y)=$ $(x-y)^{2}$. Define $f: X \rightarrow X$ and $F: X \rightarrow C B(X)$ such that $f x=x$ and $F x=\{2 x\}$ for all $x \in X$. Suppose that the property (E-A), holds then there exists a sequence $\left\{x_{n}\right\}$ in $X$ such that for some $t \in X$ and $A \in C B(X)$

$$
\lim _{n \rightarrow \infty} f x_{n}=t \in A=\lim _{n \rightarrow \infty} F x_{n} .
$$

Then $\lim _{n \rightarrow \infty} f x_{n}=t, A=\{2 t\}$. Obviously $t$ does not belong to $A$. Thus $f$ and $F$ do not satisfy the property (E-A).

Definition 9. ([2], [13]) Let $(X, d)$ be a symmetric (semi-metric) space and maps $f: X \rightarrow X$ and $F: X \rightarrow C B(X)$ then pair $(f, F)$ is said to be compatible of type $N$ iff $f x \in F x \Rightarrow f f x \in F f(x)$.

ExAmple 3. Let $X=[1, \infty)$ with $d(x, y)=(x-y)^{2}$. Let $f: X \rightarrow X$, $F: X \rightarrow C B(X)$ such that $f(x)=2 x$ and $F x=[1,2 x+1], \forall x \in X$. Then $f x=2 x \in[1,2 x+1]=F x$ and $f f x=4 x \in[1,4 x+1]=F f x$. So it is compatible of type $(N)$. 


\section{Main results}

Our main results run as follows:

Theorem 1. Let $(X, d)$ be symmetric (semi-metric) space. That enjoys [W3] [the Hausdorffness of $\tau(d)$ ]. Let $f$ be self mapping of $X$ and $F: X \rightarrow$ $C B(X)$ such that

(i) $f$ and $F$ satisfy the property (E-A),

(ii) for all $x \neq y \in X$,

$$
\begin{aligned}
H(F x, F y)<\max \left\{d(f x, f y), \frac{1}{2}[d(f x, F x)+d(f y, F y)],\right. & \left.\frac{1}{2}[d(f y, F x)+d(f x, F y)]\right\} .
\end{aligned}
$$

If $f(X)$ be $d$-closed $(\tau(d)$-closed) subset of $X$, then $f$ and $F$ have a point of coincidence.

Proof. Firstly, one needs to note that a sequence $\left\{x_{n}\right\}$ in a semi-metric space $(X, d)$ converges to a point $x$ in $\tau(d)$ iff $d\left(x_{n}, x\right) \rightarrow 0$. To substantiate this suppose $x_{n} \rightarrow x$ and $\varepsilon>0$. Since $S(x, \varepsilon)$ is a neighborhood of $x$, there exists $U \in \tau(d)$ such that $x \in U \subset S(x, \varepsilon)$. Since $x_{n} \rightarrow x$ there is a $m \in N$ (the natural number) such that $x_{n} \in U \subset S(x, \varepsilon)$ for $n \geq m$. So $d\left(x_{n}, x\right)<\varepsilon$ for $n \geq m$ that is $d\left(x_{n}, x\right) \rightarrow 0$.

The converse part is obvious in view of the definition of $\tau(d)$.

Now in view of (i), there must exists a sequence $\left\{x_{n}\right\}$ in $X$ with $t \in X$ and $A \in C B(X)$ such that

$$
\lim _{n \rightarrow \infty} f\left(x_{n}\right)=t \in A=\lim _{n \rightarrow \infty} F x_{n} .
$$

Since $f(X)$ is $d$-closed, so every convergent sequence of points of $f(X)$ has a limit in $f(X)$, therefore

$$
\lim _{n \rightarrow \infty} f\left(x_{n}\right)=f(a) \quad \text { for some } a \in X .
$$

Thus from (3.2)

$$
t=f a \in A=\lim _{n \rightarrow \infty} F x_{n}
$$

We claim $f a \in F a$.

If it is not so, i.e. if $f a \neq F a$, then in view of (3.1) we get

$$
\begin{aligned}
& H\left(F x_{n}, F a\right)<\max \left\{d\left(f x_{n}, f a\right), \frac{1}{2}\left[d\left(f x_{n}, F x_{n}\right)+d(f a, F a)\right],\right. \\
& \left.\frac{1}{2}\left[d\left(f a, F x_{n}\right)+d\left(f x_{n}, F a\right)\right]\right\} .
\end{aligned}
$$

Taking the limit as $n \rightarrow \infty$, we obtain 


$$
\begin{aligned}
H(A, F a) \leq \max \left\{d(f a, f a), \frac{1}{2}[d(f a, A)+\right. & d(f a, F a)], \\
& \left.\frac{1}{2}[d(f a, A)+d(f a, F a)]\right\} .
\end{aligned}
$$

Since $f a \in A$ so $d(f a, F a)<\max \left\{\frac{1}{2} d(f a, F a), \frac{1}{2} d(f a, F a)\right\}<d(f a, F a)$ which is a contradiction. Hence $f a \in F a$. This shows that $f a$ is a point of coincidence for $f$ and $F$.

EXAMPLE 4. Let $X=[1, \infty)$ with $d(x, y)=(x-y)^{2}$, and maps $f: X \rightarrow X$, $F: X \rightarrow C B(X)$ such that $f(x)=x^{2}$ and $F x=[1, x+1] \forall x \in X$. Then $f$ and $F$ satisfy the property (E-A) for the sequence $\left\{1+\frac{1}{n}\right\}$ and

$$
\begin{aligned}
& H(F x, F y)<d(f x, f y) \\
& \leq \max \left\{d(f x, f y), \frac{1}{2}\left[d(f x, F x)+d(f y, F y), \frac{1}{2}[d(f y, F x)+d(f x, F y)]\right\} .\right.
\end{aligned}
$$

Thus, all conditions of Theorem 1 are satisfied and $1=f 1 \in F 1$.

Since non compatible hybrid pair $(f, F)$ satisfy property (E-A), we have the following:

Corollary 1. Let $(X, d)$ be symmetric (semi-metric) space that enjoys [W3] (the Hausdorffness of $\tau(d)$ ). Let $f$ be a self mapping and $F: X \rightarrow$ $C B(X)$ such that

(i) $f$ and $F$ are non compatible,

(ii) for all $x \neq y \in X$,

$$
\begin{aligned}
H(F x, F y)<\max \left\{d(f x, f y), \frac{1}{2}[d(f x, F x)+d(f y, F y)],\right. & \left.\frac{1}{2}[d(f y, F x)+d(f x, F y)]\right\} .
\end{aligned}
$$

If $f(X)$ be a closed subset of $X$, then $f$ and $F$ have a coincidence point.

Theorem 2. Let $(X, d)$ be a symmetric (semi-metric) space enjoys [W3]. Let $f$ be a self mapping of $X$ and $F: X \rightarrow C B(X)$ such that

(i) $f$ and $F$ satisfy the property (E-A),

(ii) for all $x \neq y \in X$,

$$
\begin{aligned}
H(F x, F y)<\max \left\{d(f x, f y), \frac{1}{2}[d(f x, F x)+d(f y, F y)],\right. & \left.\frac{1}{2}[d(f y, F x)+d(f x, F y)]\right\},
\end{aligned}
$$

(iii) $f f(v)=f v$ for $v \in C(f, F)$, set of coincidence points of $f$ and $F$,

(iv) $(f, F)$ is pair of compatible of Type $(N)$. 
If $f(X)$ be $d$-closed $(\tau(d)$-closed) subset of $X$. Then $f$ and $F$ have a common fixed point.

Proof. In view of Theorem 1 and (iii) $f f v=f v$ for $v \in C(f, F)$.

Let $f a$ does not belong to $F f(a)$. Since $f a \in F a$ and $F a \neq F f(a)$

$$
\begin{aligned}
H(F a, F f a)<\max \left\{d(f a, f f a), \frac{1}{2}[d(f a, F a)+d(f f a, F f a)],\right. & \left.\frac{1}{2}[d(f f a, F a)+d(f a, F f a)]\right\} .
\end{aligned}
$$

Since $(f, F)$ is pair of compatible type $(N)$, so $t=f a \in F a \Rightarrow f f a \in F f a$, so $d(f a, F f a)<\frac{1}{2} d(f a, F f a)$, which is a contradiction.

So $f a \in F f a \Rightarrow f f a \in F f a$ [from (iii) $f f v=f v] \Rightarrow f a=f f a \in F f a$, hence $t=f t \in F t$. Thus $t$ is common fixed point of $f$ and $F$.

EXAmple 5. Consider $X=[0,1]$ equipped with the symmetric $d(x, y)=$ $(x-y)^{2}$. Define $f: X \rightarrow X$ and $F: X \rightarrow C B(X)$ as follows.

$$
\begin{gathered}
f x= \begin{cases}1-x, & \text { if } 0 \leq x \leq \frac{1}{2}, \\
0, & \text { if } \quad \frac{1}{2}<x \leq 1,\end{cases} \\
F x= \begin{cases}{\left[0, \frac{1}{2}\right],} & \text { if } \quad 0 \leq x \leq \frac{1}{2}, \\
{\left[\frac{1}{4}, \frac{3}{4}\right],} & \text { if } \quad \frac{1}{2}<x \leq 1 .\end{cases}
\end{gathered}
$$

Clearly $f(X)=\{0\} \cup\left[\frac{1}{2}, 1\right]$ is d closed in $X$. The pair $(f, F)$ enjoys the property (E-A) as for the sequence $\left\{\frac{1}{2}-\frac{1}{n}\right\} \subset[0,1]$, we have

$$
\lim _{n \rightarrow \infty} f\left(\frac{1}{2}-\frac{1}{n}\right)=\frac{1}{2} \in \lim _{n \rightarrow \infty} F\left(\frac{1}{2}-\frac{1}{n}\right)=\left[0, \frac{1}{2}\right] \in[0,1] .
$$

By routine calculation one can show that the contractive condition (3.1) holds for every $x \neq y \in X$. Also notice that the

$$
f\left(\frac{1}{2}\right)=\frac{1}{2} \in F\left(\frac{1}{2}\right)=\left[0, \frac{1}{2}\right] \Rightarrow f f\left(\frac{1}{2}\right)=\frac{1}{2} \in\left[0, \frac{1}{2}\right]=F f\left(\frac{1}{2}\right)
$$

i.e. $(f, F)$ is pair of compatible type $(N)$.

Since the topology induced by $d$ is usual on $[0,1]$, it will be Hausdorff and therefore condition [W3] is naturally satisfied. Thus all the conditions of Theorem 2 are satisfied and $\frac{1}{2}$ is the unique common fixed point of $f$ and $F$.

Here one needs to note that $d$ is not a metric as

$$
d(0,1)>d\left(0, \frac{1}{2}\right)+d\left(\frac{1}{2}, 1\right)
$$


Thus, all the available metrical common fixed point theorems can not be used in the content of this example, notice that both the maps $f$ and $F$ are discontinuous at the unique common fixed point " $\frac{1}{2}$ ".

Corollary 2. Let $(X, d)$ be symmetric (semi-metric) space that enjoys [W3] (the Hausdorffness of $\tau(d)$ ). Let $f$ be a self mapping on $X$ and $F: X \rightarrow C B(X)$ such that

(i) $f$ and $F$ satisfy the property (E-A),

(ii) for all $x \neq y \in X$,

$$
\begin{array}{r}
H(F x, F y)<\max \left\{d(f x, f y), \frac{1}{2}[d(f x, F x)+d(f y, F y)],\right. \\
\left.\frac{1}{2}[d(f y, F x)+d(f x, F y)]\right\},
\end{array}
$$

(iii) $f f(v)=f v$ for $v \in C(f, F)$, set of coincidence points of $f$ and $F$,

(iv) $f$ is injective (one-one) mapping.

If $f(X)$ be $d$-closed $(\tau(d)$ closed) subset of $X$, then $f$ and $F$ have a common fixed point.

Proof. From (iv) $f$ is an injective (one-one) mapping. Therefore $f v=f u \Rightarrow$ $v=u$. From condition (iii) $f f(v)=f v$ for $v \in C(f, F)$. So $f f(v)=f v \Rightarrow$ $f v=v$ and for $f v \in F v \Rightarrow f f(v) \in F f v$, i.e. $(f, F)$ is pair of compatible of type $(N)$.

Definition 10. [10] Let $X$ be a symmetric (semi-metric) space. Let $f, g: X \rightarrow X$ and $F, G: X \rightarrow C B(X)$. The pair maps of $(f, F)$ and $(g, G)$ are said to satisfy the common property (E-A) if there exist two sequences $\left\{x_{n}\right\},\left\{y_{n}\right\}$ in $X$, some $t$ in $X$ and $A, B$ in $C B(X)$ such that

$$
\lim _{n \rightarrow \infty} F x_{n}=A, \lim _{n \rightarrow \infty} G y_{n}=B, \lim _{n \rightarrow \infty} f x_{n}=\lim _{n \rightarrow \infty} g y_{n}=t \in A \cap B .
$$

EXAMPLE 6. Let $X=[1, \infty)$ with the symmetric $d(x, y)=(x-y)^{2}$. Let $f, g: X \rightarrow X$ and $F, G: X \rightarrow C B(X)$ defined by $f(x)=2+\frac{x}{3}, g(x)=2+\frac{x}{2}$, and $F(x)=[1,2+x], G(x)=\left[3,3+\frac{x}{2}\right]$ for all $x \in X$. Consider the sequence $\left\{x_{n}\right\}=\left\{3+\frac{1}{n}\right\},\left\{y_{n}\right\}=\left\{2+\frac{1}{n}\right\}$. Clearly,

$$
\begin{gathered}
\lim _{n \rightarrow \infty} F x_{n}=[1,5]=A, \quad \lim _{n \rightarrow \infty} G y_{n}=[3,4]=B, \\
\lim _{n \rightarrow \infty} f x_{n}=\lim _{n \rightarrow \infty} g y_{n}=3 \in A \cap B .
\end{gathered}
$$

Therefore, $(f, F)$ and $(g, G)$ are said to satisfy the common property (E-A).

Theorem 3. Let $f, g$ be two self-maps of the symmetric (semi-metric) space $(X, d)$ and let $F, G$ be two maps from $X$ to $C B(X)$ such that 
1. $(f, F)$ and $(g, G)$ satisfy the common property (E-A),

2. for all $x \neq y \in X$,

$$
\begin{aligned}
H(F x, G y)<\max \left\{d(f x, g y), \frac{1}{2}[d(f x, F x)+d(g y, G y)],\right. \\
\left.\frac{1}{2}[d(f x, G y)+d(g y, F x)]\right\} .
\end{aligned}
$$

If $f X$ and $g X$ are closed subsets of $X$, then

(a) $f$ and $F$ have a coincidence point;

(b) $g$ and $G$ have a coincidence point;

(c) $f$ and $F$ have a common fixed point provided that pair $(f, F)$ is compatible of type $(N)$ and $f f v=f v$ for $v \in C(f, F)$;

(d) $g$ and $G$ have a common fixed point provided that pair $(g, G)$ is compatible of type $(N)$ and $g g v=g v$ for $v \in C(g, G)$;

(e) $f, g, F$ and $G$ have a common fixed point provided that both (c) and (d) are true.

Proof. Since $(f, F)$ and $(g, G)$ satisfy the common property (E-A), there exist two sequences $\left\{x_{n}\right\},\left\{y_{n}\right\}$ in $X$ and $u \in X, A, B \in C B(X)$ such that

$$
\lim _{n \rightarrow \infty} F x_{n}=A, \quad \lim _{n \rightarrow \infty} G y_{n}=B, \quad \lim _{n \rightarrow \infty} f x_{n}=\lim _{n \rightarrow \infty} g y_{n}=u \in A B .
$$

By virtue of $f X$ and $g X$ being closed, we have $u=f v$ and $u=g w$ for some $v, w \in X$. We claim that $f v \in F v$ and $g w \in G w$. Indeed, condition (3.1) implies that

$$
\begin{aligned}
& H\left(F x_{n}, G w\right)<\max \left\{d\left(f x_{n}, g w\right), \frac{1}{2}[\right.\left.d\left(f x_{n}, F x_{n}\right)+d(g w, G w)\right], \\
&\left.\frac{1}{2}\left[d\left(f x_{n}, G w\right)+d\left(g w, F x_{n}\right)\right]\right\} .
\end{aligned}
$$

Taking the limit as $n \rightarrow \infty$, we obtain

$$
\begin{aligned}
& H(A, G w) \\
& <\max \left\{d(f v, g w), \frac{1}{2}[d(f v, A)+d(g w, G w)], \frac{1}{2}[d(f v, G w)+d(g w, A)]\right\} \\
& =\frac{1}{2} d(g w, G w)<d(g w, G w) .
\end{aligned}
$$

Since $g w=f v \in A$, it follows from the definition Hausdorff semi-metric that $d(g w, G w)=H(A, G w)<d(g w, G w)$ which implies that $g w \in G w$. 
On the other hand, by condition (3.1) again, we have

$$
\begin{aligned}
H\left(F v, G y_{n}\right)<\max \left\{d\left(f v, g y_{n}\right),\right. & \frac{1}{2}\left[d(f v, F v)+d\left(g y_{n}, G y_{n}\right)\right], \\
& \left.\frac{1}{2}\left[d\left(f v, G y_{n}\right)+d\left(g y_{n}, F v\right)\right]\right\} .
\end{aligned}
$$

Similarly, we obtain

$$
d(f v, F v)=H(f v, B)<d(f v, F v) .
$$

Hence $f v \in F v$. Thus, $f$ and $F$ have a coincidence point $v, g$ and $G$ have coincidence point $w$. This ends the proofs of part (a) and part (b).

Furthermore, by virtue of condition (c), we obtain $f f v=f v$ and $f f v \in$ $F f v$. Thus $u=f u \in F u$. This proves (c). A similar argument proves (d). Then (e) holds immediately.

Acknowledgement. The authors thank very much the referee for his valuable comments and suggestions.

\section{References}

[1] M. Amari, D. El. Moutawakil, Some new common fixed point theorems under strict contraction conditions, J. Math. Anal. Appl. 270 (2002), 181-188.

[2] M. Amari, D. El. Moutawakil, Common fixed points under contractive conditions in symmetric spaces, Appl. Math. E-Notes 3 (2003), 156-162.

[3] Seong-Hoon Cho, Gwang-Yeon Lee, Jong-Sook Bae, On coincidence and fixed-point theorems in symmetric spaces, Fixed Point Theory Appl. Vol. 2008, article ID 562130, 9 pages, doi:10.1155/2008/562130.

[4] M. Imdad, J. Ali, L. Khan, Coincidence and fixed points in symmetric spaces under strict contractions, J. Math. Anal. Appl. 320 (2006), 352-360.

[5] G. Jungck, Compatible mappings and common fixed points, J. Math. Math. Sci. 9 (1986), 771-779.

[6] G. Jungck, Common fixed points for non-continuous non-self maps on non-metric spaces, Far East. J. Math. Sci. 4 (1996), 199-225.

[7] G. Jungck, B. E. Rhoades, Fixed points for set-valued functions without continuity, Indian J. Pure Appl. Math. 29 (1998), 227-238.

[8] T. Kamran, Coincidence and fixed points for hybrid strict contractions, J. Math. Anal. Appl. 299 (2004), 235-241.

[9] H. Kaneko, S. Sessa, Fixed point theorems for compatible multivalued and single valued mappings, Int. J. Math. Math. Sci. 12 (1989), 257-262.

[10] Y. Liu, J. Wu, Zhixiangli, Common fixed points of single-valued and multi-valued maps, Int. J. Math. Math. Sci. 19 (2005), 3045-3055.

[11] R. P. Pant, V. Pant, Common fixed points under strict contractive conditions, J. Math. Anal. Appl. 248 (2000), 327-332.

[12] R. P. Pant, V. Pant, K. Jha, Note on common fixed points under strict contractive conditions, J. Math. Anal. Appl. 274 (2002), 879-880. 
[13] N. Shahazad, T. Kamran, Coincidence points and R-weakly commuting maps, Arch. Math. (Brno) 37 (2001), 179-183.

[14] P. K. Shrivastava, N. P. S. Bawa, P. Singh, Coincidence theorems for hybrid contraction II, Soochow J. Math. 26(04) (2000), 411-421.

[15] W. A. Wilson, On semi-metric spaces, Amer. J. Math. 53 (1931), 361-373.

\author{
Yogesh Kumar Vijaywar \\ DEPARTMENT OF MATHEMATICS \\ GOVT. J.S.T.P.G. COLLEGE \\ BALAGHAT M.P., INDIA, 481001 \\ E-mail: yogesh_vijaywar@yahoo.com \\ N. P. S. Bawa \\ DEPARTMENT OF MATHEMATICS \\ GOVT. SCIENCE COLLEGE \\ REWA, M. P., INDIA, 481006 \\ E-mail: promptbawa@yahoo.com \\ P. K. Shrivastava \\ DEPARTMENT OF MATHEMATICS \\ GOVT. J.S.T.P.G. COLLEGE \\ BALAGHAT M.P., INDIA, 481001 \\ E-mail: pshrivastava@yahoo.com
}

Received November 24, 2009; revised version August 9, 2010. 